\title{
Svevo: la coscienza della lontananza
}

\section{Massimo Rizzante}

\section{Narrare opus est}

Il destino di un ebreo che si avverta scrittore è quello di narrare. Potremmo dire, interpretando Buber, che anche narrare è un incontro e un guardare che cosa veramente sta difronte, un sein wahres Gegenüber. Il nostro essere nella realtà dovrebbe, secondo Buber, essere vissuto in termini di Io-Tu e non piú di Io-Esso. Ovvero, la riduzione soggettivistica della realtà, che secondo la tradizione filosofica occidentale può nascere solo dopo che, attraverso un processo di astrazione intellettuale, si è costituito l'oggetto su cui indagare, troverebbe la sua reale possibilità e quindi verità solo quando il soggetto entrasse con il mondo in un rapporto dialogante, l'Io-Tu appunto. L'impossibilità di delimitare il nostro io rappresentato rende la nostra esperienza spezzata, con Benjamin nello stato della povertà.

Narrare, per chi lo senta come destino, sarà allora tentare una circolarità d'esperienza che è anche esistenziale complementarietà del nostro essere con il mondo. Fare in modo che l'esperienza del narrare non resti 'solo' letteratura, è la necessità a cui lo scrittore ebreo deve sottostare, pena la perdita della sua differenza, che è sí ancestrale struttura psichica della rassegnazione, ma soprattutto sguardo 'illimitato' sul mondo, cognizione religiosa delle cose.

La differenza che lo scrittore Svevo vive come scrittore ebreo si farà, nelle sue pagine, segreta, perché inconciliabile con un presente che sente lontano da essa e che lo porterà alla dissimulazione, o meglio alla rappresentazione di una dissimulazione: il personaggio inetto. Svevo riconosce la sua lontananza da quella tradizione ebraica di un narrare come esperienza comune e affettiva, propria di quell'ebraismo chassidico d'oriente non pervenuto a nessuna assimilazione storica con la società occidentale, e dove il modello della conoscenza e per la conoscenza è il dialogo e la preghiera; ma egli non rinuncerà a sperimentare un personaggio che nella sua inadeguatezza al vivere smaschera sottilmente un vero e proprio allontanaQUADERNI d' italianistica Volume VIII, No. 2, 1987 
mento degli uomini dai propri bisogni, i quali, non piú rintracciabili, si sono resi 'ideali.' Come dice Giorgio Voghera, parlando degli scrittori ebrei triestini della grande stagione letteraria del primo novencento, nel suo libro Gli anni della psicanalisi “. . . se c'è uno spirito ebraico che ha influito sui letterati di cui parliamo, questo non può essere se non lo spirito degli ebrei occidentali ormai staccatisi dalle forme di vita e dalla spiritualità-alle volte altissima — del ghetto. ..." e ancora "... l'ebreo occidentale è spesso spiritualmente piú vicino al non ebreo che all'ebreo orientale. .." (137).

La saggezza chassidica, mai disgiunta dalla compassione per gli uomini, che permette a Rabbi Pinhas de Koretz, un grande maestro del XVIII secolo, di rispondere ad un suo giovane allievo che stava perdendo la sacralità della vita “. . . va et etude la Torah. La Torah est le seul reméde; elle l'a toutjours ete. Elle contient toutes les réponses. Elle est la réponse. L'aurais-tu oublié?" (9) non è piú dicibile per lo scrittore ebreo semi-assimilato,che non avverte piú il 'giogo della Torah' e non conosce risposte sagge, perché esse implicano un'esperienza con il mondo, un dialogo con le cose che, agli inizi del XX secolo, è stato interrotto.

In Svevo, inoltre, non si avrà né la mitizzazione dell'unità di valori ostjudish, incarnata dal Giobbe di Roth-mitizzazione che è soprattutto legame con la tradizione ebraico-orientale-né la superdeterminazione della colpa ontologica dell'esistere di Kafka.

Vogliamo dire: sono diverse 'resistenze' all'assimilazione. Roth vive, fin nelle sue fibre fisiche, il depauperamento dell'esperienza e la sua desacralizzazione nell'ebreo piccolo borghese assimilato, giungendo non solo a rompere con la tradizione, come dice Magris “. . . perché è la storia occidentale, divenuta ormai tradizione, a degradare i valori transindividuali. .." ma, come lo stesso autore prosegue "... rompe, del resto, persino con la tradizione del regno biblico. . ." rimanendo, cosí, legato soltanto all'ebraismo della diaspora. Tutta la sua narrativa sarà, cosí, essa stessa una lunga ricerca senza centro, un esilio da un invisibile Heimat.

Per Kafka non si tratta di una vera e propria resistenza, ma di un'alienazione dall'ebraismo puro e incontaminato del ghetto. La colpa di non essere nell'ebraismo, ma ai margini della sua conoscenza, produrrà un linguaggio, come dice Gargani, 'costitutivo' e non piú 'descrittivo.' Kafka richiederà alla sua scrittura una trasfor- 
mazione che diventi opposizione e trasgressione ad una legge sacra, che lui vede burocraticizzata. Di piú, la sua stessa scrittura sarà una difesa a oltranza del suo esilio, del suo sapersi muto nella conoscenza della ragione ma, come dice Freschi, "sulla soglia della Terra promessa" (149).

Con Kafka si vive l'irriconoscibilità di una condizione, del Geist ebraico, l'essere cioè nella colpa della solitudine; non piú nella legge che comunica e fa comunicare, ma nella lingua che, come dice egli stesso negli ultimi suoi anni, è "un'amante perduta." La sua esperienza della scrittura sarà, forse, allora l'indecisione, il limite del concetto e dell'immagine, il limitare il concetto nell'immagine, facendo cosí il suo linguaggio 'costitutivo,' come affermavamo innanzi, assunta l'impraticabilità della tradizione, la quale, se non fosse stata perduta, avrebbe "descritto" il senso."

Svevo, anch'egli scrittore ebreo semi-assimilato, vive la sua 'resistenza' all'ordine piccolo borghese della società occidentale, potremmo dire, in forma di scepsi. Ovvero, la dissimulazione di un personaggio inetto, di cui prima parlavamo, non è rintracciabile essenzialmente nello Selbsthass, nell'odio di sé, che l'ebreo prova per la sua mai completa integrazione e appartenenza; odio di sé che lo stesso Debenedetti pone come incipit alla comprensione della narrativa sveviana, ma, soprattutto, in quello che vorremmo chiamare 'sentimento di una lontananza.' La lontananza non ha luogo, come dice Magris; essa è vissuta come sentimento senza nome, che produce un nascondimento, un personaggio che ostenta la sua dissimulazione e che rivela la sua inadeguatezza alla società in cui vive. Ma proprio questo 'nascondimento' di sé del personaggio sveviano svela l'allontanamento degli altri, degli altri pesonaggi dalla propria origine. Origine che è, essenzialmente, vero bisogno e non idealizzazione di esso. Ed è qui, crediamo, che quel 'sentimento di lontananza,' proprio dell'ebreo che 'resiste,' seppure attraverso una dissimulazione, all'assimilazione, si vela di una veste filosofica borghese.

È l'educazione schopenhaueriana di Svevo che, intrecciandosi con la constatazione ebraica di una secolarizzazione dei rapporti sociali e amorosi, pone il personaggio nel luogo di una volontà interrotta; nell'aspirazione incompiuta che si farà via via nostalgico destino, sogno amoroso, ironia e, infine, resoconto di una mancata redenzione di sé. ${ }^{2}$ La differenza, perciò, che abbiamo detto inconciliabile 
con un presente dominato dalla pura lotta del vivere dello scrittore ebreo triestino e del suo personaggio ha un viso bifronte. Essa ha un'ascendenza dalla metafisica borghese che nega l'autenticità dei rapporti umani fondati puramente sui bisogni-anche se fa di questi ultimi necessità e virtú-e una matrice ebraica che riconosce la sua lontananza dal messaggio originario, ma si fa destino e, solo apparentemente, nell'inettitudine si contraddice; rivelandosi nascostamente, invece, istanza critica, accusa di una caduta di valori. La differenza dello scrittore e del suo personaggio sarà, allora, da una parte, necessità di una lontananza propria dell'ebreo e, dall'altra, diventerà un sentirsi differente, un ritenere la propria superiorità inaccessibile, pur riconoscendo migliaia di meriti negli altri; un'aspirazione da letterato, insomma, che gli farà sognare la vita piuttosto che possederla, letterarizzarla, infine.

L'inettitudine è questa lontananza da questo mondo borghese, è questo senso borghese di superiorità che vuole redimere con la letteratura il mondo, conoscendo-si pensi a Zeno Cosini-l'ironia del tutto.

\section{La nostalgia di una lontananza-L'amoroso sogno}

Incentrando per ora il discorso, seppur brevemente, sui primi due romanzi di Svevo, potremmo dire che essere letterati, filosofi significa, cosí per Alfonso come per Emilio, volere il proprio destino piú rischioso; non fermarsi all'evidenza, anzi, non guardarla neppure, affondare i colpi d'ala al di là del divenire, cosí privo di veri possessi. La loro posizione, però, è quella dell'inerzia, dell'attesa che vive come vizio il suo eterno ripetersi. il dissimulare di Alfonso un interesse al dialogo in casa Maller, quando, invece, già l'entrata e l'incontro con Santo-il servo-mostrano una serie di divieti; l'impossibilità di Alfonso di essere uno di loro e il senso di impotenza che lo coglie e gli interrompe il discorso, gettandolo nell'aspettazione del momento buono per parlare che, anche se giunge, non lo soddisfa; e, in Senilità, la necessità di Emilio di non essere quasi mai se stesso per non perdere il suo amore, per non perdere il suo sogno di Ange; sono tutti momenti di una tentazione, quella di distruggere il rinvio, che si fanno, irrimediabilmente, gesti di un vizio: quello della lontananza dalla realtà.

Il personaggio sveviano ${ }^{3}$ ha bisogno di trasfigurare le cose, di 
amare con Schopenhauer ciò che è oltre l'apparenza; ma, cosí facendo, sente il suo destino lontano, ebraicamente allontanato dall'origine, nell'attesa di un vero possesso di se stesso e delle cose. In Una vita da una lontananza vissuta come destino nostalgico-Alfonso e l'alienazione cittadina, l'ordine e l'efficienza del lavoro bancario a cui egli non riesce a sottostare, il ricopiare che non lo esprime, la grettezza di casa Lanucci che è figlia del puro sforzo per sopravvivere, la conoscenza e l'amore che non riempiono, neppure con il materiale possesso, il vuoto della continua aspirazione ideale-al ritrovamento dei veri valori. Il ritorno di Alfonso al centro. Il villaggio è la sensazione di ritrovarsi nel proprio elemento, anche se egli è lí per attendere ad una morte. La lunga agonia della madre è il prezzo di una rigenerazione del figlio che si era allontanato dal mondo dei padri. L'attesa, qui nel luogo d'origine, è un'attesa dolorosa ma con il segno dell'accettazione-come dicono gli ebrei-delle creature che accettano la creazione (Buber L'eclissi di Dio). È quasi rito e liturgia, allora, anche il delirio e lo stato di semi-morte di Alfonso dopo la morte della madre.

Scontare lo stato di coscienza è un'esperienza terribile; comporta un'espiazione sacrificale, una 'promenade' infernale. La rigenerazione non è nuova generazione, ma totale accettazione di Alfonso del suo distacco dall'origine, suggellato dal bacio materno che egli riceve in sogno, il bacio della madre morta che gli lascia il dolore e la compassione per la vita. ${ }^{4}$

La partenza per la città è sotto il segno apparente "dell'uscita dalla puerizia" (Una vita 309), una delle tante, come egli stesso rimedita, ma nel profondo è già lontananza dalla vita, dalla lotta organica e irresistibile che essa innesca con ogni individuo. il dramma della vita non può essere ammirato, deve essere tradotto in una parte. Alfonso vive l'impossibilità di questa traduzione e si rassegna al sogno che non rinuncia di credere la vita un sogno giovanile. Perché morire nella lotta, in un duello senza speranze con il giovane Maller proprio adesso che "il sogno non lo aveva posseduto giammai cosí interamente?" (Una vita 401). La lontananza dalla vita che il bacio materno, in sogno, suggella definitivamente è anche affezione al sogno, dopo il distacco dall'origine.

In Senilità, poi, la nostalgia del ritorno viene a mancare e la vera giovinezza, essendosi la nostalgia trasformata in vera e propria co- 
scienza di una lontananza, è solo di colui che amorosamente si attacca al sogno, a quel suo sapersi irrimediabilmente lontano da ogni giovinezza, intesa, non solamente come salute, ma come perdita dell'uomo dei suoi bisogni. La senilità che viene dopo l'esasperazione di un'attesa di salute e salvezza è proprio il luogo della lontananza che si è fatta, anche, malattia organica e di pensiero. Emilio aspira al possesso delle cose vicine, ma, invece, constata che l'unica verità, l'unico possesso che gli è concesso è quella lontananza, quella capacità di sognare che è l'unica giovinezza.

Il sogno, che si era impossessato di Alfonso e gli aveva fatto compiere l'ultimo atto, si fa per Emilio amorosa verità. Il possesso puro e "bestiale" non gli è concesso. Egli è nato non per la lotta ma per il sogno e per continuare a sognare. Angiolina che, con la sua salute e spregiudicatezza, sembra spezzare il ritmo onirico della vita di Emilio, non fa altro che consustanziarlo, lo allontana di piú dalla vita. La donna incarna il sogno di Emilio ma non lo fa esplodere, perché il suo concedersi non è un darsi, è un possesso del corpo liberato dalla mente. Ella è giovane cosí nel corpo come nella mente, e non vive che questa sua sensuale giovinezza biologica cosí vicina alla pura necessità, ma lontana dalla profonda salute di chi riesce a "pensare e a piangere." Perciò Emilio sognerà sempre una Angiolina che non esiste ed ogni possesso sarà fittizio, una sottrazione, nel suo senso ambivalente.

Sarà la sorella Amalia, con il suo 'destino grigio,' con la sua lenta agonia, ma anche con la sua intelligenza e purezza, il referente inconscio di Emilio e del suo impossible sogno. Egli vuole un'Angiolina che sappia piangere e soffrire come Amalia, sappia, nel silenzio, amare e consumarsi in quella tensione. L'agonia della sorella e la sua morte coincideranno, cosí, con l'abbandono di Angiolina; e nel tempo la stessa memoria coagulerà in un'unica immagine le due figure femminili. Il sogno irrealizzabile nella realtà è, nella memoria letteraria di Emilio, corretto e quasi reso mitico. La donna sarà per sempre "amante" e "pensosa." proprio come può essere scritta e non vissuta, proprio come la si è voluta che fosse e non come è stata realmente. Ed è cosí che la letteratura, questa dolorosa finzione, vince, come dice lo stesso Svevo in Senilità, l'inettitudine dell'uomo alla vita, seppure essa stessa resti, ed è questo il vero dolore, nel luogo di una volontà interrotta, necessariamente interrotta dalla morte. 
Sentimento di una lontananza in Alfonso, coscienza di una lontananza che sa il proprio destino immutabilmente incompiuto e, perciò, affetto dal rinvio in Emilio.

Nei due personaggi non si avrà ribellione proprio perché il loro destino li ha allontanatị da ciò che veramente sono, producendo cosí un nascondimento, che è svelamento, ebraicamente, di una secolarizzazione dei rapporti umani; nascondimento che genera il sogno ed il rinvio continuo, nella realtà, di un atto decisivo.

Narrare opus est. Narrare è necessario, allora, per vincere l'inettitudine, perché il sogno si trasformi in parola e possa essere compreso o frainteso, comunque con dolore, vissuto.

In questo senso, il personaggio sveviano è pure indice, come molti altri personaggi della 'fin de siècle,' della problematizzazione dell'individuo e della sua atomizzazione nel linguaggio, che ha portato l'uomo a monologare, a trasformare l'esistenza in sogno.

La necessità della scrittura non è, perciò, momento dell'esperienza; nasce, invero, dall'impossibilità di un dialogo con le cose, nasce come farmaco per l'esperienza, definendo, cosí, una frattura tra uomo e scrittore, tra l'inettitudine dell'uomo moderno e la superiorità fittizia, grottesca dello scrittore.

Il personaggio sveviano, ripetiamo, è la rappresentazione di questa dicotomia, che è superiorità dello scrittore che vive tale superiorità come senso di inferiorità negli atti, perché gli atti hanno perso quella che Baioni, parlando di Kafka in Ebraismo e Mitteleuropa, chiama "radice celeste," la sacralità necessaria perché l'uomo li riconosca propri.

Alfonso è condannato al sogno ed Emilio all'attesa che il suo sogno si incarni, anche se, alla fine, capirà che il suo sogno, o il suo sogno fattosi parola, sono le uniche armi che egli possiede per redimere la realtà. È la condanna al sogno, che significa lontananza dalla storia, che conduce ad una precoce senilità. Essa diventa il luogo di quella lontananza da dove si può, peraltro, solo sognare un possesso della storia, consapevoli del fatto che quest'ultima si è già impossessata di noi, lasciandoci la possibilità del sogno o l'invenzione letteraria.

Non vi è salute nella storia, quindi. I sani sono gli assimilati, quei personaggi sveviani che vivono nella sensualità della vita, coloro che sono nella storia con la solarità propria di chi è vicino e cammina 
con il proprio destino. Ma la loro non è vera salute. L'inettitudine del personaggio sveviano svela l'allontanamento di sé dalla sua salute illusoria, e si fa 'superiore inferiorità' dello scrittore-impiegato che, lontano dal proprio destino, si attacca al sogno, al sogno della scrittura che è malattia sí, ma amorosa.

\section{L'ironia del senso}

Con Zeno altre caratteristiche, già presenti negli altri due romanzi, vengono alla luce e fatte, per cosí dire, esplodere. Con la Coscienza di Zeno la critica ai valori della società borghese si farà quasi metodo, seppure in forma di ricordo e di tragica prospettiva. L'allontanamento del personaggio dal suo destino ha provocato l'ironia del senso, l'allontanamento da ogni senso.

Zeno rappresenta, ancora, il bisogno di scrivere la vita che non è possibile puramente vivere, essendo essa una malattia essenziale, una imperfezione che conduce l'uomo alla lotta per il suo compimento, che, peraltro, è solo costruzione di un ordine puntualmente distrutto. Ma qui, nel terzo romanzo, a colui che conosce la malattia della vita e il suo destino nato da tale convinzione, non è il sogno che resta. Mentre, cioè, nei romanzi precedenti, il sogno era l'attività che trasfigurava o addirittura negava la vita, e il personaggio 'inetto' non poteva che attaccarsi amorosamente alla sua rappresentazione e alla sua malattia, nella Coscienza di Zeno l'atteggiamento, tipicamente ebraico, dell'autodifesa, della sapiente ironia che conosce tutte le amarezze che porta in seno, fa parlare il protagonista sui sogni, sulla loro utilità per la vita, anche se 'il metodo dei sogni' porta con sé, nella stessa traduzione, la sua morte. E lo scetticismo, ancora una volta ebraico di una razionalizzazione totale dell'uomo, lo porterà ad affermare che i sogni, o meglio, la predisposizione ad essi, si conclude sempre nella loro invenzione.

Potremmo affermare che, per un processo metonimico, il sogno necessario alla vita del personaggio letterato, viene fatto esplodere nell'ironia della sua impossibile traduzione e descrizione da parte di Zeno, il vecchio uomo di lettere che ha compreso il nihilismo di quella posizione. La consistenza della letteratura diventerà, allora, lo scriversi. Finito il sogno del letterato inizia una letteratura fatta di memorie e invenzione.

Zeno che si sottopone alla cura psicanalitica ha accettato la pro- 
pria malattia come incurabile e ricerca, sogghignando di ogni arte medica dell'animo umano, le possibilità future nel proprio passato. Riconosce la propria differenza di destino e la sua lontananza da esso, ma non sogna, meglio, non si attacca al suo sogno; ironizza sulla stessa scienza dei sogni.

Diversamente dai due precedenti romanzi, dove la differenza era lontananza del personaggio dalle sue origine e dove quest'ultima era dissimulata nell'inettitudine cronica e nella precoce senilità, ora, nella Coscienza di Zeno, quella stessa differenza-ciò per cui l'uomo è differente al di là delle differenze-si è fatta consapevolezza ironica e meccanismo di difesa; e ancora, critica, non piú sotto forma di svelamento attraverso il nascondimento del sé originario del personaggio, ma, seppur velata, diretta. Zeno, cioè, è ancora, anche se in modo relativamente diverso, il personaggio che porta con sé la distonia esistenziale dell'ebreo occidentale, consapevole della lontananza delle sue origini e che fa di questa lontananza il luogo e la dimora da dove mettere a nudo e in crisi la pura lotta del vivere, la volontà di dominio, il mondo della mometa che sempre si può cambiare.

È proprio nelle pagine dedicate al padre-significativamente alla morte del padre-che è possibile constatare l'indice di questa consapevole lontananza del figlio che, ebraicamente, rifiuta l'assimilazione ad una società che il padre sembra aver fatto finta di non vedere, ma nella quale ha certamente operato e guadagnato. Quest'ultimo è il peccato che Zeno non vorrebbe mai commettere ma che, inevitabilmente, ha dovuto sempre commettere. Il padre-lo dice testualmente piú volte-è il se stesso che rifiuta, ma anche qualcosa di piú. Suo padre è anche il se stesso piú profondo che non riesce a dire la parola all'uomo e schiaffeggia il figlio, il se stesso che ha dovuto assimilarsi. Ciò che resta è una parola non detta e il rimorso per quella parola che avrebbe reso, finalmente, alla luce "la sua grande esperienza," come dice il padre a Zeno (689). Zeno afferra, forse, che l'esperienza del padre non si è veramente contaminata con l'esperienza del mondo in cui ha vissuto. Il padre, che vedeva dappertutto una realtà immutabile ed un mondo immobile, aveva, forse, salvato la sua differenza originaria, ma la morte, come sempre, era giunta troppo presto. Il rimorso di Zeno è quello del figlio che è giunto troppo tardi a riconoscere il padre, che ha creduto troppo che la serietà del padre fosse ridicola e, perciò, non gli si è mai avvicinato abbastanza 
ed ha giocato, al solito, al rinvio. Qui è la monade generatrice di ogni altro dramma di Zeno. Zeno riderà piú forte del mondo e il suo riso filosofico e selvaggio potrà anche inclinare a suo favore il 'clinamen' della vita, ma resterà sempre il riso amaro, comune a molti ebrei della sua generazione, di colui che non si è ricongiunto amorosamente con la tradizione. ${ }^{5}$ Il tempo della Creazione è immutabile $^{6}$ e il sentimento di chi vive il tempo come immutabilità è il sentimento di chi accetta la Creazione. L'ispirazione religiosa, che fa dire al padre di possedere una grande esperienza da comunicare è quel "sentimento d'intelligenza" dell'uomo che accetta le cose e ammette la loro trasparenza; di chi, insomma, vede la legge dietro ogni divenire, dell'ebreo che si è salvato dalla secolarizzazione. ${ }^{7} \mathrm{Di}$ versamente per Zeno l'ispirazione religiosa del padre è oggetto di studi: "tant'è vero che s'indusse a parlarmene perché io gli avevo raccontato di essermi occupato delle origini del Cristianesimo" (691). Il momento che fraternamente li accomuna è anche il momento del piú vero distacco.

Per l'ebreo che riconosce la lontananza delle sue origini e non si è ricongiunto al mondo dei padri, la religione è un problema, il tempo è un problema. Milan Kundera ha scritto una volta: “. . . il tempo del romanzo di Kafka è il tempo di un'umanità che ha perduto la sua continuità con l'umanità, di un'umanità che non sa piú nulla e non ricorda piú nulla e abita in città che non hanno nome e le cui strade sono strade senza nome o con un nome diverso da quello che portavano ieri, perché un nome è una forma di continuità con il passato e le persone che non hanno passato sono persone senza nome" (169-70).

Se Kafka si aggirava nella Praga, che il suo amico Max Brod definiva la città del male, profeta di un mondo senza memoria Svevo, nella Coscienza di Zeno, approda, a suo modo, ad una soluzione; fa, a suo modo, della memoria un atto che riconosce il passato il quale, però, attraverso la scrittura, che si sa comunque letteratura, viene ritradotto, corretto. Il suo non è un 'sibi scribēre,' uno scrivere della propria vita e sulla propria vita, ma, essenzialmente, un ripensamento evolutivo; un ripensare, cioè, il passato che, nella practica letteraria, si trasforma e si radicalizza fino all'invenzione.

Zeno si scrive, abbiamo detto. Giunto ad una certa età, vuole veder 'chiaro' il proprio passato. Si sottopone ad una cura psica- 
nalitica senza crederci troppo; la sua malattia la conosce fin troppo bene, è una convinzione carica di anni e di destino. Egli sa anche perfettamente che non è possibile estirparla: essa ha una genealogia troppo profonda. Ciò che si può fare, allora, è illudersi che il sogno e l'inerzia procurino il dono della rievocazione. Ma Zeno è un letterato, incanutito, con problemi di peso e di cuore, ma eterno studente; egli non riesce a ricordare con precisione, l'ordine gli è sempre sembrato un uso rassicurante dell'ambizione umana, e, poi, la lingua che adopera, si conosce, è piuttosto uno scalpello che una lima. Egli inventa. La memoria di Zeno è una memoria pratica, proprio in quanto ha perduto il suo passato (coscienza, ancora una volta, della lontananza) e si compie per atti volontari che, diremmo, redentivi. Egli non attende, con Proust, il senso del presente da un'interruzione di esso che permette l'esplosione del passato cosí com'era e che ritorna carico di un'esperienza non vissuta, e perciò colma di felicità. Zeno ha una 'volontà della memoria' che, nella scrittura, diventa metafora di una redenzione del passato attraverso una correzione di esso.

Il tempo della scrittura diventa cosí, ebraicamente, il tempo della redenzione, la quale, però, deve essere intesa in senso probematico, in quanto si conosce la lontananza delle proprie origini e la difficoltà, quindi, di un riconoscimento del passato attualizzabile.

Il tempo in cui Zeno vive non è il tempo immutabile del padre, il tempo della Creazione nel quale le cose annunciano il loro essere innocente e nel quale anche il loro dissolversi necessario si concepisce come eterno compimento del passato. Il tempo che 'ritorna,' quando Zeno si abbandona alla scrittura terapeutica, è un passato offuscato dal presente che sorge "imperioso" e annulla la ridicola pretesa del protagonista di sperimentare da vicino una memoria che veramente ricordi. Zeno si addormenta di un sonno profondo e, seppure durante quel sonno qualche cosa di importante era stato intravvisto, al risveglio è già stato dimenticato, "perduto per sempre."

Nel Preambolo al romanzo, infatti, Zeno informato dal suo buon manuale di psicanalisi che una pratica della memoria quotidiana consente molte possibilità di ricordare la propria infanzia, si mette subito al 'lavoro.' Dopo qualche esitazione, vede di fronte a sé l'immagine di un bambino in fasce che sembra appartenergli da vicino, ma che subito ricosce per il figlio della cognata appena nato e che lui aveva 
visto di recente. Zeno, che conosce nell'intimo la minacciosa sentenza di malattia che cova nell'uomo fin dalla sua venuta al mondo, esclama: "Come fare? è impossibile tutelare la tua culla. Nel tuo seno-fantolino-si va facendo una combinazione misteriosa. Ogni minuto che passa vi getta un reagente. Troppe probabilità di malattia vi sono per te, perché non tutti i tuoi minuti possano essere puri. Eppoi fantolino sei consanguineo di persone ch'io conosco. I minuti che passano possono anche essere puri, ma certo tali non furono tutti i secoli che ti prepararono" (651).

Il tempo del ricordo è un tempo che ha subíto e subisce la storia, proprio come il pensiero subisce la sua alienazione, il suo essere di fronte a Zeno eppure estraneo alla sua volontà di rappresentarlo. Ma il pensiero che non si configura nel presente e una memoria che non è figura del passato sono ancora propri di un personaggio ebreo che non trova risarcimento morale nella storia, che appunto la subisce perché egli ne è stato tolto ed è consapevole che la sua assimilazione non ha significato, intimamente, radicamento postumo, ma soprattutto sradicamento e dispersione. Ecco, allora, che la scrittura, lo scrivere di Zeno ciò che Zeno fa, diventa la possibilità redentiva del tempo, il poter ripensare la storia secondo una legge astorica, qual è la pratica letteraria. Ma tale possibilità redentiva, che infine è la scrittura, oltre ad essere problematica, come abbiamo detto prima, è anche ironica, perché cosciente che il suo atto è pur sempre un ripensamento e mai un riconoscimento. La scrittura vive cosí il tempo del rilancio, della scommessa, che vuol dire, per Zeno, vivere per rinviare il senso perduto della sua origine.

\section{Università di Urbino}

\section{NOTES}

1 crf. Gargani e Freschi 149: "La diaspora assume un segno invisibile, l'ebreo occidentale irriconoscibile porta dentro di sé il suo nome, il suo alfabeto sacro."

2 Relativamente a quest'ultima affermazione, facciamo riferimento al cosiddetto quarto romanzo sveviano di cui parla Contini ma del quale non si tratterà in questa sede, poiché esula dagli scopi del presente lavoro.

$3 \mathrm{Si}$ fa riferimento, ovviamente, al protagonista dei primi due romanzi. Il discorso sarà, infatti, sensibilmente diverso per quanto riguarda la Coscienza di Zeno. 
4 Si legge infatti, in Una vita 304; "Con movimento istintivo egli aveva chiuso gli occhi per isolarsi. Era sua madre. Prima che ella giungesse al suo letto egli la vide e vide il suo sorriso soddisfatto di trovarlo tanto quieto. Ella si chinò su di lui e lo baciò, ma giusto sulla cavità dell'orecchio. Egli sentí un acuto dolore come se dentro qualche cosa fosse scoppiato e si svegliò."

5 Si veda nella Coscienza di Zeno 690-91: "Oggi che scrivo, dopo di aver avvicinata l'età raggiunta da mio padre, so con certezza che un uomo può avere il sentimento di una propria altissima intelligenza che non dia altro segno di sé fuori di quel suo forte sentimento. Ecco: si dà un forte respiro e si accetta e si ammira tutta la natura com'è e come, immutabile, ci è offerta: con ciò si manifesta la stessa intelligenza che volle la Creazione intera."

6 Cfr. la nota precedente.

$7 \mathrm{Cfr}$. La coscienza di Zeno 691: "Da mio padre è certo che nello ultimo istante lucido della sua vita, il suo sentimento d'intelligenza fu originato da un'improvvisa ispirazione religiosa. ..."

\section{OPERE CONSULTATE}

A.A.V.V., (1983), Ebraismo e Mitteleuropa. Atti del convegno promosso dall'istituto per gli studi mitteleuropei di Gorizia. Brescia: Shakespeare e C., 1983.

BENJAMIN, W. Angelus Novus. Torino: Einaudi, 1982.

BUBER, M. I racconti dei Chiassidim. Milano: Garzanti, 1979.

. L'eclissi di Dio. Milano: Comunità, 1983.

CONTINI, G. Il quarto romanzo di Svevo. Torino: Einaudi, 1980.

GARGANI, A. e M. FRESCHI. Kafka, oggi. Napoli: Guida, 1984.

KUNDERA, M. Il libro del riso e dell' oblio. Milano: Bompiani, 1980.

MAGRIS, C. Lontano da dove. Torino: Einaudi, 1977.

SVEVO, I. Senilitd. Milano: Mondadori, 1985.

. La coscienza di Zeno. Milano: Mondadori, 1985.

TOULMIN, S.e A. JANIK. La grande Vienna. Milano: Garzanti, 1984.

VOGHERA, G. Trieste nei miei ricordi. Pordenone: Studi Tesi, 1980.

. Quaderno d'Israele. Pordenone: Studi Tesi, 1986.

WIESEL, E. Contre la Mélancolie. Paris: Seuil, 1981.

. L'ebreo errante. Firenze: La Giuntina, 1983. 